\title{
Majority-Vote Model on Scale-Free Hypergraphs
}

\begin{abstract}
T. Gradowski*, And A. KRAWIECKi
Faculty of Physics, Warsaw University of Technology, Koszykowa 75, PL-00662 Warsaw, Poland

Majority-vote models on scale-free hypergraphs are investigated by means of numerical simulations with different variants of system dynamics. Hypergraphs are generalisations of ordinary graphs in which higher order of social organisation is included by introducing hyperedges corresponding to social groups, connecting more than two nodes. In the models under study, opinions of agents (two-state spins) placed in nodes are updated according to a probabilistic rule with control parameter representing social noise. The probability of a single spin flip depends on the average opinion within only one social group (hyperedge) the agent belongs to. This introduces an intermediate level of social interactions, in contrast with the case of networks, where the opinion of an agent usually depends on the average opinion of all neighbours. In all cases under consideration a second-order phase transition to a state with an uniform opinion was found as a function of the social noise, with the critical value of the control parameter and the critical exponents depending on the hypergraph topology and details of the system dynamics (node or hyperedge update).
\end{abstract}

DOI: 10.12693 /APhysPolA.127.A-55

PACS: 89.75.Hc, 64.60.Cn, 05.10.Ln, 05.50.+q, 64.60.F-

\section{Introduction}

The majority vote (MV) model with two states [1] (for review see [2]) is a nonequilibrium stochastic model of the opinion formation. In this model, agents (two-state spins) update their opinions at discrete time steps following the opinion of the majority of their neighbours (or that of the majority of a selected subset of their neighbours) with certain probability $1-q, 0 \leq q \leq 1 / 2$, which controls the degree of randomness in the system dynamics. The update rules are not associated with any Hamiltonian and the model does not obey the detailed balance. According to [3] such non-equilibrium stochastic spin systems with up-down symmetry on regular lattice can exhibit second-order phase transition and belong to the universality class of the corresponding equilibrium Ising model. In fact, the MV model on regular two- $[1,4]$ and three-dimensional [5] lattices was shown to exhibit a second-order phase transition with the critical exponents belonging to the universality class of the corresponding equilibrium Ising model; it was also shown to follow the mean-field (MF) critical behaviour above a finite upper critical dimension [6]. In contrast, the critical exponents for the MV model on various sorts of complex networks [7-13], e.g., small-world [9], scale-free (SF) [10, 11], Apollonian [12], Archimedean [13], etc., networks are clearly different than those for the corresponding equilibrium Ising model; therefore, in these cases the MV model does not belong to the universality class of the corresponding Ising model. Recently, there has been growing interest in models of interacting systems on various generalised complex networks (for review see [14]). Following this interest, in this paper we study the critical properties of the MV model on complex hypergraphs,

*corresponding author; e-mail: tomgrad@if.pw.edu.pl which generalise the concept of complex networks in the following way: in networks pairs of nodes are connected by edges, while in hypergraphs groups of more than two nodes are connected by hyperedges [15]. With respect to models of opinion formation, hyperedges can connect different groups of people the agent belongs to and interacts with, e.g., family members, co-workers, friends, discussion groups, etc. As an example we consider complex SF hypergraphs in which the distribution of hyperdegrees of nodes (number of hyperedges $k_{i}$ attached to a given node $i$ ) obeys a power law $P\left(k_{i}\right) \propto k_{i}^{-\alpha}$.

\section{Evolving scale-free hypergraphs}

SF hypergraphs can be constructed as evolving hypergraphs using a preferential attachment algorithm which generalises that for evolving SF networks [16-18]. The algorithm starts with $n$ nodes connected by $m_{h}$ hyperedges, each of which connects all initial $n$ nodes. In each step of the construction $m$ new nodes are added to the hypergraph $(1 \leq m \leq n-1)$ which are then connected by $m_{h} \geq 1$ new hyperedges to $m_{h}$ sets of $n-m$ randomly chosen existing nodes according to the following preferential attachment rule: The probability to attach a new hyperedge to the existing node $i$ is proportional to the hyperdegree $k_{i}$ of this node, $p_{i}=k_{i}\left(\sum_{j} k_{j}\right)^{-1}$, where the summation runs over all existing nodes. It should be emphasised that all $m$ new nodes are connected by each new hyperedge to $n-m$ nodes which may, but need not be connected by another hyperedge or hyperedges. The process stops after a desired number of nodes $N$ in the hypergraph is reached. The resulting hypergraph is $\mathrm{SF}$, with the distribution of the hyperdegrees of nodes obeying a power law, $P(k) \propto k^{-\alpha}, \alpha=1+\frac{n}{n-m}$, for $k \geq m_{h}$, with the exponent $\alpha>2$. The exponent does not depend on $m_{h}$, which modifies only the normalisation constant and the range of the distribution. It should be noted that the above-mentioned model allows multiple 
connections of the same nodes with different hyperedges (in particular, the first $n$ nodes are connected by $m_{h} \geq 1$ hyperedges).

\section{The model}

The MV model on a hypergraph consists of a set of spin-like variables $\sigma$, representing opinions of agents. Each spin can take one of two values: +1 or -1 . Spins are located in nodes of a complex hypergraph.

The critical properties of the model are investigated by numerical simulations. We introduce two types of dynamics: hyperedge-update and node-update, both focusing on the formation of the opinion in groups. The hyperedgeupdate dynamics is as follows. One hyperedge $e$ is randomly selected and the majority opinion is checked in this very hyperedge. Next the value of each spin $\sigma_{i}$ in the selected hyperedge is flipped with probability

$$
w_{i}(\sigma)=\frac{1}{2}\left(1-(1-2 q) \sigma_{i} \operatorname{sgn}\left(\sum_{j \in e} \sigma_{j}\right)\right),
$$

where the $\operatorname{sgn}(\ldots)$ is the majority opinion in the group (hyperedge) $e$, equal zero in case of lack of majority and the sum runs over all nodes attached to the hyperedge $e$. The control parameter $q$ can be interpreted as a social temperature and plays a role similar to that of temperature in equilibrium systems. The closer to zero the $q$ is, the greater the probability of agreement with the local majority. Next, the procedure is repeated until all the hyperedges in the model are updated. Thus, one time step of the simulation consists in updating all hyperedges and every hyperedge is updated once per one time step, only the sequence is random. The opinion of the agent in each node is updated as many times as the number of hyperedges the node is attached to (the hyperdegree of the node).

The node-update dynamics is as follows. One node $i$ and one of its hyperedges $e$ are randomly selected. Next, the majority opinion in the selected hyperedge is determined and the $\sigma_{i}$ is updated with probability given by Eq. (1). One time step of the dynamics consists in updating all nodes in random order.

It should be noted that in both kinds of dynamics, the state of the spin $\sigma_{i}$ is included in the sum in Eq. (1), which to some extent models the agents' supportiveness (devotion to the opinion they currently hold).

The main difference between the proposed dynamics rules is, that in the case of hyperedge-update dynamics the agent's opinion in each node in every time step is updated as many times, as the number of hyperedges attached to this node. One can say that each agent interacts with all his/her neighbours, which makes the dynamics more MF like. On the other hand in the case of node-update dynamics, the agent, independent of its hyperdegree, interacts with only one group of its neighbours (hyperedge). This situation resembles that on regular low-dimensional hypercubic lattices where the number of neighbours is finite and rather small; nevertheless, in our model different time steps the agent can interact with different groups of neighbours.

\section{Methods of simulations and analysis}

Simulations for both variants of dynamics are performed in the same way. The SF hypergraph with the parameters $n, m, m_{h}$ is generated using the algorithm described in Sect. 2. For each value of $q$, we assume uniform initial conditions (all spins set to 1 ). Next $T_{\text {trans }}$ time steps of simulation are performed to overcome the transient $\left(T_{\text {trans }}=10^{4}\right.$ for the hyperedge-update dynamics and $10^{5}$ for the node-update dynamics) and $T$ steps of actual survey ( $T=10^{4}$ for the hyperedge-update and $10^{5}$ for the node-update dynamics). All steps are repeated $20-60$ times for each value of $q$ with different realisations of the SF hypergraph.

To study the critical behaviour the variable $m$ is defined, which is the average opinion in the system,

$$
m=\frac{1}{N} \sum_{i=1}^{N} \sigma_{i}
$$

Next, the magnetisation $M$, susceptibility $\chi$ and the fourth-order Binder cumulant $U_{4}$ are examined,

$$
\begin{aligned}
& M(q)=[\langle|m|\rangle]_{a v}, \\
& \chi(q)=N\left[\left(\left\langle m^{2}\right\rangle-\langle|m|\rangle^{2}\right)\right]_{a v}, \\
& U_{4}(q)=1-\left[\frac{\left\langle m^{4}\right\rangle}{3\left\langle m^{2}\right\rangle^{2}}\right]_{a v} .
\end{aligned}
$$

The angle brackets $\langle\ldots\rangle$ denote average over $T$ time steps and square brackets $[\ldots]_{a v}$ denote averaging over different realisations of the hypergraph.

The above quantities are expected to obey finite size scaling (FSS) relations. We assume that the form of these relations is analogical to those for interacting systems on complex (e.g. SF) networks [19], i.e.,

$$
\begin{aligned}
& M=N^{-\beta / \nu} f_{m}\left(N^{1 / \nu}\left(q-q_{c}\right)\right), \\
& \chi=N^{\gamma / \nu} f_{\chi}\left(N^{1 / \nu}\left(q-q_{c}\right)\right), \\
& q^{*}(N)-q_{c} \propto N^{-1 / \nu} .
\end{aligned}
$$

The critical noise value $q_{c}$ can be obtained from the intersection point of the Binder cumulant functions for different sizes $N$ of the particular hypergraph. Next, from Eq. (3a,b) the exponents $\beta / \nu$ and $\gamma / \nu$, respectively, can be determined. Furthermore, Eq. (3c) can be used to calculate the exponent $1 / \nu$ using the value $q^{*}(N)$ for which the susceptibility $\chi$ of the model on the hypergraph with $N$ nodes has a maximum value. Finally, it is checked if the obtained exponents fulfill the hyperscaling relation,

$$
2 \frac{\beta}{\nu}+\frac{\gamma}{\nu}=D_{e f f}
$$

where the effective dimensionality $D_{\text {eff }}=1$ is expected in the case of systems on complex networks or hypergraphs which do not have any particular spatial dimension [19]. 


\section{Results}

In Fig. 1 the dependence of the Binder cumulant $U_{4}$ on the noise parameter $q$ is shown for exemplary SF hypergraph for different values of $N$. Second-order phase transition is clearly visible, with $U_{4}$ values decreasing from

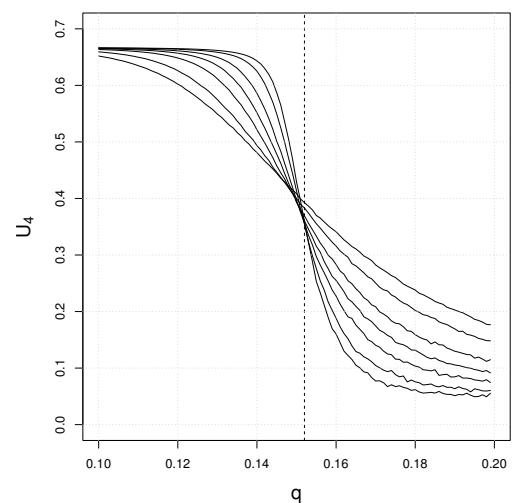

Fig. 1. The Binder cumulant $U_{4}$ as a function of the noise parameter $q$ for the exemplary SF hypergraph with $n=3, m=1, m_{h}=3$ and with node-update dynamics. Results were obtained for $N=10^{3}, 2 \times 10^{3}, 5 \times 10^{3}$, $10^{4}, 2 \times 10^{4}, 5 \times 10^{4}, 10^{5}$. The critical noise $q_{c} \approx 0.152$ was obtained as the curves intersection point.
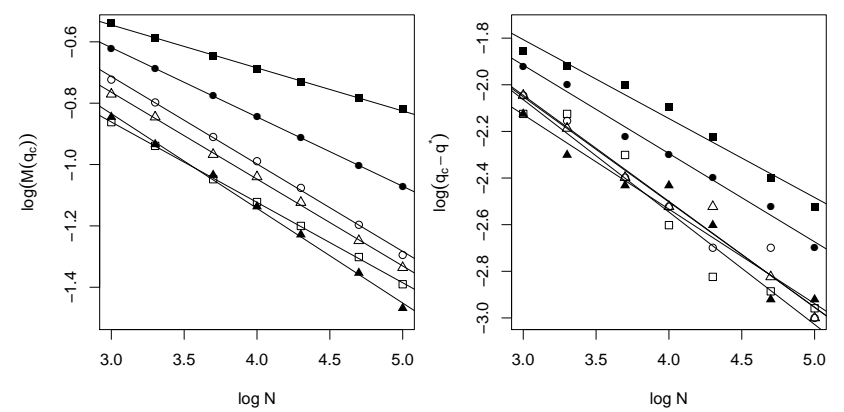

Fig. 2. Log-log plot of the magnetisation $M$ and susceptibility $\chi$ as a function of the number of nodes $N$ for SF hypergraphs with: $n=3, m=2, m_{h}=3$ (squares); $n=3, m=1, m_{h}=3$ (circles); $n=4$ $m=3, m_{h}=3$ (triangles). Empty symbols correspond to the hyperedge-update dynamics, solid symbols represent the node-update dynamics. Each curve was obtained at the critical noise value $q_{c}$ (see Table). Solid lines show the best fits with the slopes $-\beta / \nu$ and $\gamma / \nu$.

$2 / 3$ to 0 while passing the critical noise value $q_{c}$. In principle from the analysis of the Binder cumulant the critical noise value $q_{c}$ can be determined, at which curves for all values of $N$ intersect. In practice (even for equilibrium models) the curves usually do not intersect exactly at one point and $q_{c}$ can be only estimated as the limit of the intersection points of the $U_{4}$ curves for increasing $N$. In agreement with Eqs. $(3 \mathrm{a}, \mathrm{b})$, at $q=q_{c}$ the magnetisation and susceptibility scale as $M \propto N^{-\beta / \nu}$ and $\chi \propto N^{-\gamma / \nu}$, respectively, as shown in Fig. 2. From the dependence $M(N)$ and $\chi(N)$ at $q=q_{c}$ the critical exponents $\beta / \nu$ and $\gamma / \nu$ are obtained, which are summarised in Table for SF hypergraphs with different topologies and dynamics.

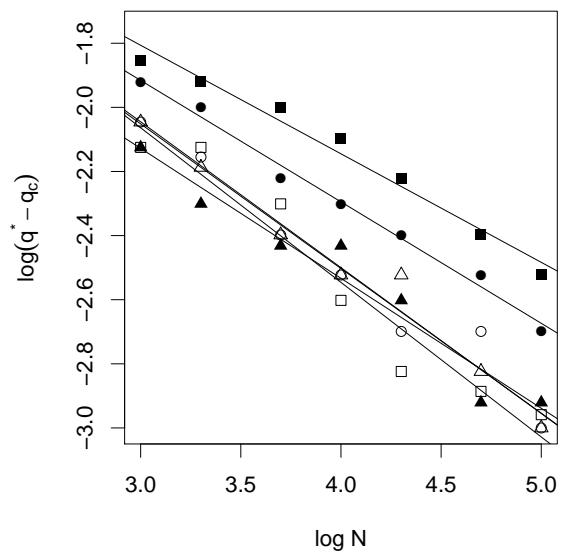

Fig. 3. Displacement of the point of maximum susceptibility $q^{*}$ from $q_{c}$ vs. the number of nodes $N$ for different SF hypergraphs and dynamics (see Fig. 2 for details). Solid lines show the best fits with the slope $-1 / \nu$. The accuracy of the slope estimation is confined due to finite resolution of $q$ in simulations.
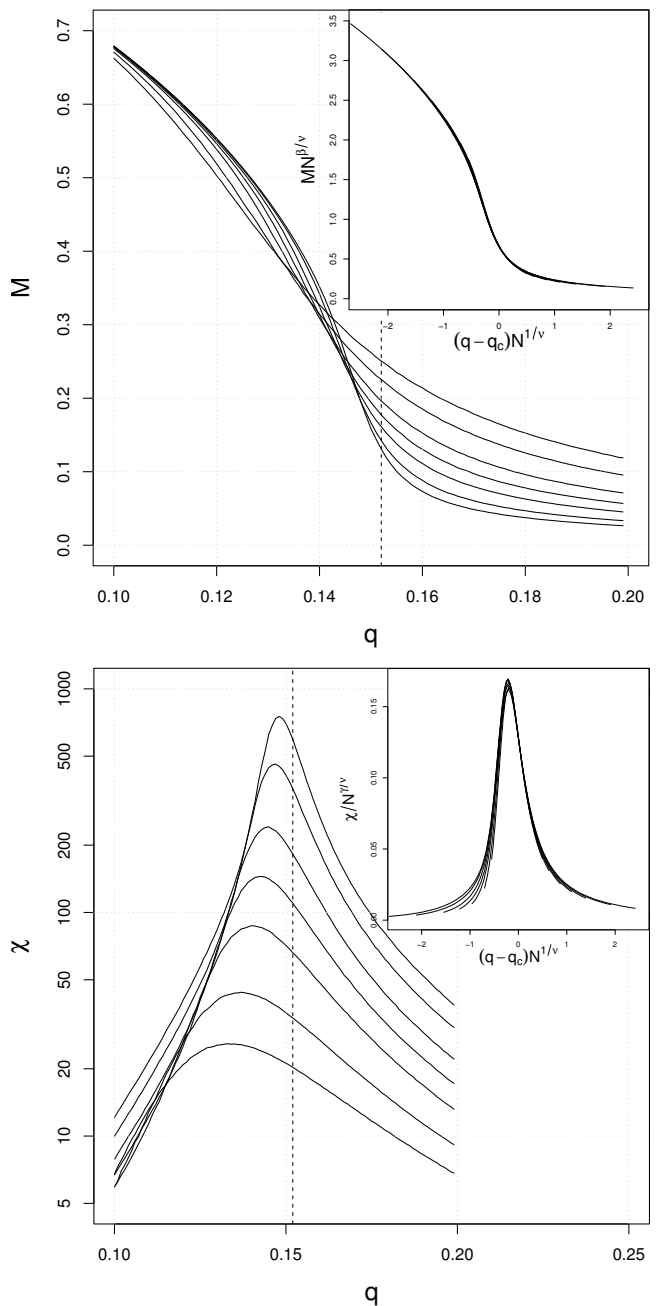

Fig. 4. Magnetisation $M$ and susceptibility $\chi$ as functions of the noise parameter $q$ for exemplary SF hypergraph (see Fig. 1 for details). Dashed lines in both plots show the critical value of noise $q_{c}$. Rescaled results with the critical exponents from Table are shown in the insets. 
According to Eq. (3c) it is expected that the displacement of the point of maximum susceptibility $q^{*}$ from $q_{c}$ scale as $N^{-1 / \nu}$. This can be seen in Fig. 3, although the accuracy of estimation of the exponent $1 / \nu$ is limited due to finite resolution of $q$ in simulations. In Fig. 4 the magnetisation and susceptibility are plotted as functions of $q$. The decreasing displacement of the maximum of the sus- ceptibility is observed while increasing the size $N$. Using the critical exponents from Table, the rescaled values of $M$ and $\chi$ are plotted (Fig. 4, insets). In accordance with Eq. (3a,b), in both cases the data collapse into one curve, which confirms the correctness of the obtained exponents. Moreover, the exponents $\beta / \nu, \gamma / \nu$ fulfill well the hyperscaling relation (4), with $D_{\text {eff }} \approx 1$, as expected.

TABLE

The critical noise $q_{c}$ and the critical exponents for different hypergraphs for both described types of dynamics.

\begin{tabular}{c|c|c|c|c|c|c|c|c|c|c}
\hline \hline \multirow{2}{*}{ Hypergraph } & \multicolumn{4}{|c|}{ Hyperedge-update } & \multicolumn{5}{c}{ Node-update } \\
\cline { 2 - 10 } & $q_{c}$ & $\beta / \nu$ & $\gamma / \nu$ & $1 / \nu$ & $D_{\text {eff }}$ & $q_{c}$ & $\beta / \nu$ & $\gamma / \nu$ & $1 / \nu$ & $D_{\text {eff }}$ \\
\hline$n=3, m=1, m_{h}=1, \alpha=2.5$ & 0.158 & 0.26 & 0.48 & 0.46 & 1.00 & 0.117 & 0.14 & 0.74 & 0.33 & 1.02 \\
$n=3, m=1, m_{h}=3, \alpha=2.5$ & 0.166 & 0.26 & 0.49 & 0.56 & 1.01 & 0.152 & 0.14 & 0.73 & 0.34 & 1.01 \\
$n=3, m=1, m_{h}=10, \alpha=2.5$ & 0.167 & 0.26 & 0.48 & 0.49 & 1.00 & 0.162 & 0.15 & 0.72 & 0.29 & 1.02 \\
$n=3, m=2, m_{h}=3, \alpha=4$ & 0.101 & 0.28 & 0.47 & 0.44 & 1.03 & 0.075 & 0.29 & 0.46 & 0.44 & 1.04 \\
$n=4, m=2, m_{h}=1, \alpha=3$ & 0.145 & 0.31 & 0.42 & 0.39 & 1.04 & 0.104 & 0.23 & 0.58 & 0.37 & 1.04 \\
$n=4, m=3, m_{h}=3, \alpha=5$ & 0.114 & 0.27 & 0.48 & 0.54 & 1.02 & 0.079 & 0.30 & 0.44 & 0.39 & 1.04 \\
$n=6, m=3, m_{h}=1, \alpha=3$ & 0.213 & 0.29 & 0.45 & 0.45 & 1.03 & 0.171 & 0.24 & 0.57 & 0.34 & 1.05
\end{tabular}

\section{Conclusions}

For both hyperedge- and node-update dynamics and for a broad range of the exponent $\alpha$ in the hyperdegree distribution the MV model on SF hypergraphs clearly presents second-order phase transition as $q$ is varied. In particular, the transition occurs at $q_{c}<1 / 2$ even for $2<\alpha<3$, in contrast with the Ising model on SF networks, which exhibits phase transition at finite temperature only for $\alpha>3$, where in the latter case $\alpha$ is the exponent in the distribution of the node degrees.

The model under study obeys the same FSS relations as those for interacting systems on complex networks, and the effective dimensionality is $D_{\text {eff }} \approx 1$, as expected.

In the case of hyperedge-update dynamics the critical exponents $\beta / \nu, \gamma / \nu$ and $1 / \nu$ weakly depend on the network topology (parameters $n, m, m_{h}$ ) and are close to their MF values for the Ising model, which are 0.25 , $0.5,0.5$, respectively. This supports the conjecture from Sect. 3 that this kind of dynamics is MF-like.

In contrast, in the case of node-update dynamics the values of $\beta / \nu, \gamma / \nu, 1 / \nu$ depend substantially on the hypergraph topology (mainly on the parameters $n, m$ ). In particular, there is significant difference between the values of the exponents for hypergraphs with $2<\alpha<3$ and for hypergraphs with $\alpha>3$. The values of the exponents $\beta / \nu, \gamma / \nu$ differ significantly from those for the equilibrium Ising model on SF networks with the same exponent $\alpha$, reported in Ref. [19]. The above result bears certain resemblance to the dependence of the critical exponents on the system dimension, and thus on the number of neighbours, in the case of the MV model on regular hypercubic lattices [6]. This supports the conjecture from Sect. 3 that the MV model with node-update dynamics is more similar to that on low-dimensional hypercubic lattices.
This paper suggests that the study of opinion formation can be extended to models on generalised complex networks (including hypergraphs), with the dynamics within social groups taken into account.

\section{References}

[1] M.J. Oliveira, J. Stat. Phys. 66, 273 (1992).

[2] C. Castellano, S. Fortunato, V. Loreto, Rev. Mod. Phys. 81, 591 (2009)

[3] G. Grinstein, C. Jayaprakash, Yu He, Phys. Rev. Lett $\mathbf{5 5}$, 2527 (1985).

[4] M.J. Oliveira, J.F.F. Mendes, M.A. Santos, J. Phys. A: Math. Gen. 26, 2317 (1993).

[5] A.L. Acuña-Lara, F. Sastre, Phys. Rev. E 86, 041123 (2012).

[6] Jae-Suk Yang, In-mook Kim, Wooseop Kwak, Phys. Rev. E 77, 051122 (2008).

[7] L.F.C. Pereira, F.G. Brady Moreira, Phys. Rev. E 71, 016123 (2005).

[8] F.W.S. Lima, A.O. Sousa, M.A. Sumuor, Physica A 387, 3503 (2008).

[9] P.R.A. Campos, V.M. de Oliveira, F.G. Brady Moreira, Phys. Rev. E 67, 026104 (2003).

[10] F.W.S. Lima, Int. J. Mod. Phys. C 17, 1257 (2006).

[11] F.W.S. Lima, Commun. Comput. Phys. 2, 358 (2007).

[12] F.W.S. Lima, A. A. Moreira, A. D. Araújo, Phys. Rev. E 86, 056109 (2012).

[13] F.W.S. Lima, Cent. Eur. J. Phys. 12, 185 (2014).

[14] S. Boccaletti, G. Bianconi, R. Criado, C.I. del Genio, J. Gómez-Gardeñes, M. Romance, I. Sendiña-Nadal, Z. Wang, M. Zanin, Phys. Rep. 544, 1 (2014).

[15] G. Ghoshal, V. Zlatić, G. Caldarelli, M.E.J. Newman, Phys. Rev. E 79, 066118 (2009).

[16] J.-W. Wang, L.-L. Rong, Q.-H. Deng, J.-Y. Zhang, Eur. Phys. J. B77, 493 (2010).

[17] Hu Feng, Zhao Hai-Xing, He Jia-Bei, Li Fa-Xu, Li Shu-Ling, Zhang Zi-Ke, Acta Phys. Sin. 62, 198901 (2013).

[18] Zhaoyan Wua, Jinqiao Duan, Xinchu Fu, App. Math. Model. 38, 2961 (2014).

[19] Hyunsuk Hong, Meesoon Ha, Hyunggyu Park, Phys. Rev. Lett. 98, 258701 (2007). 